\title{
PERSPECTIVES
}

\section{Plus vite, plus petit, plus sensible. Analyse et Séparations à l'aube du $3^{\text {ème }}$ Millénaire}

\section{Quicker, smaller, smarter. Analysis and Separations towards the $3^{\text {rd }}$ Millenium}

Antoine-Michel SIOUFFI

UMR 6516, Université Aix Marseille 3, Faculté Des Sciences de Saint Jerôme - 13397 MARSEILLE Cedex 20 Tél : 0491288584 - e-mail : Antoine-Michel.Siouffi@UFR.u-3mrs.fr

\section{Introduction}

Le téléphone portable, l'ordinateur portable, la micro caméra ne sont plus des objets d'étonnement et les électroniciens savent graver toute la puissance d'un ordinateur sur moins de $1 \mathrm{~cm}^{2}$ de silicium. Nous communiquons avec le monde entier, et on s'irrite parfois quand la communication (avec Internet, par exemple ) n'est pas instantanée .Le consommateur s'inquiète d'entendre parler de contamination accidentelle, mais ne sait pas que l'on contrôle certaines toxines au niveau de la ppt (partie par trillion). Le chronométrage des performances est effectué au millième de seconde. Les récentes affaires de dopage ont mis en lumière la rapidité et la sensibilité des méthodes de détection. Ces quelques exemples montrent le chemin parcouru depuis quelques années vers la miniaturisation, la facilité d'utilisation, le gain de performance et la vitesse de réponse. En fait, nous sommes au début d'une ère nouvelle, et les développements à venir vont envoyer les instru- ments actuels au musée. Il n'est qu'à regarder un spectrophotomètre miniaturisé pour en être convaincu (Figure 1).

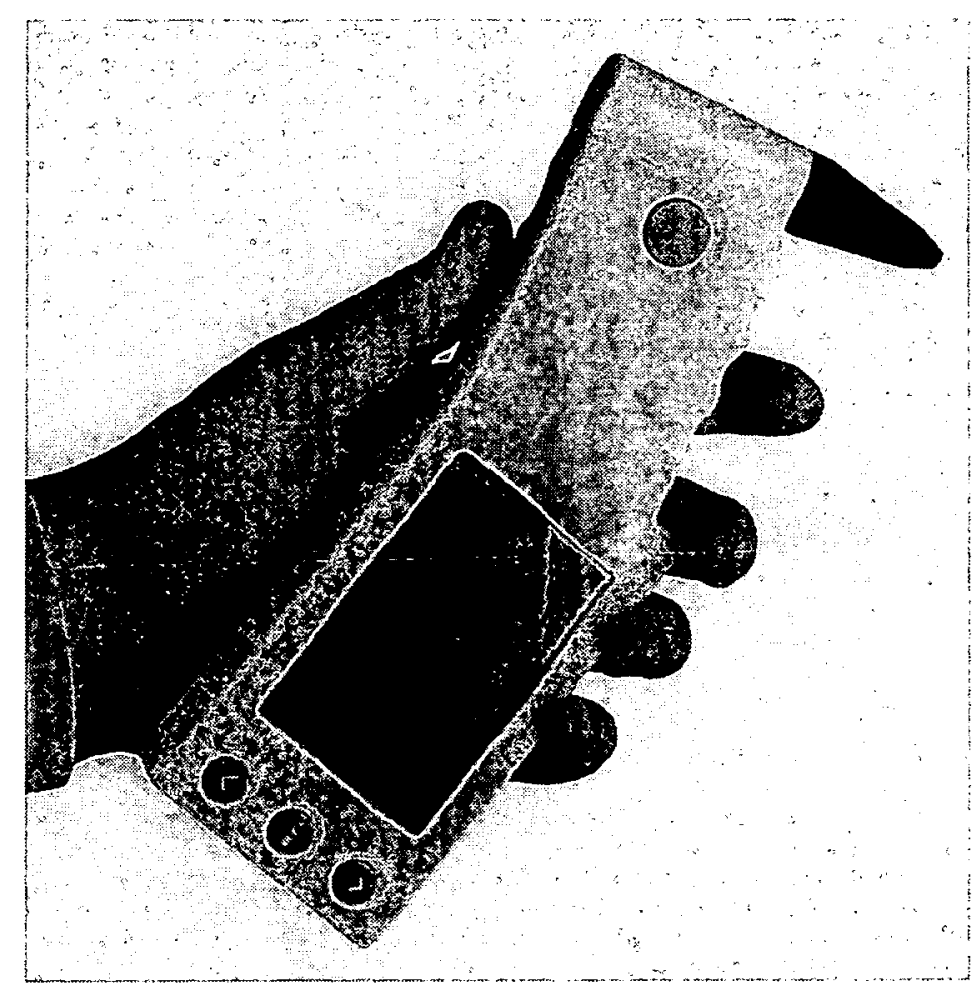

Figure 1 : Un spectrophotomètre miniaturisé. 


\section{Plus d'information, plus vite !!}

En analyse, on peut distinguer les systèmes séparatifs et les systèmes d'identification. Il faut pouvoir identifier sans ambiguïté les divers constituants préalablement séparés. La tendance actuelle et irréversible est de coupler les deux systèmes pour gagner du temps et obtenir des informations aussi complètes que possible. Ce n'est pas facile, car les matrices contenant les solutés à séparer et à identifier sont complexes (sang, urine..). Cette difficulté, liée au développement de la chimie combinatoire, a poussé les fabricants à mettre sur le marché des systèmes intégrés à haut rendement pouvant faire rapidement face à la demande. La chimie combinatoire permet d'accéder à plusieurs milliers de molécules en un temps très court. Il faut pouvoir traiter cette masse énorme de composés pour savoir très vite quels sont ceux qui ont un avenir. L'analyse est réalisée soit en perfectionnant des appareils existant soit en en créant de nouveaux.

Les systèmes séparatifs génèrent des pics dont la largeur est de l'ordre de la seconde ou moins. En chromatographie gazeuse (GC), les progrès de l'électronique et la qualité des matériaux ont permis le développement de la chromatographie rapide (Fast GC), dans laquelle on peut réaliser des programmations de température de $200^{\circ}$ en 20 secondes. On comprend pourquoi la stabilité des phases est cruciale. Les vitesses de gaz vecteur sont de l'ordre de $220 \mathrm{~cm} / \mathrm{sec}$, les colonnes sont plus courtes et de diamètre plus restreint. Les injecteurs à contrôle cryogénique permettent d'introduire des bandes de 5 à $10 \mathrm{~ms}$. Quelques exemples de séparations ultra rapides (1) sont reportés dans les figures 2 et 3.

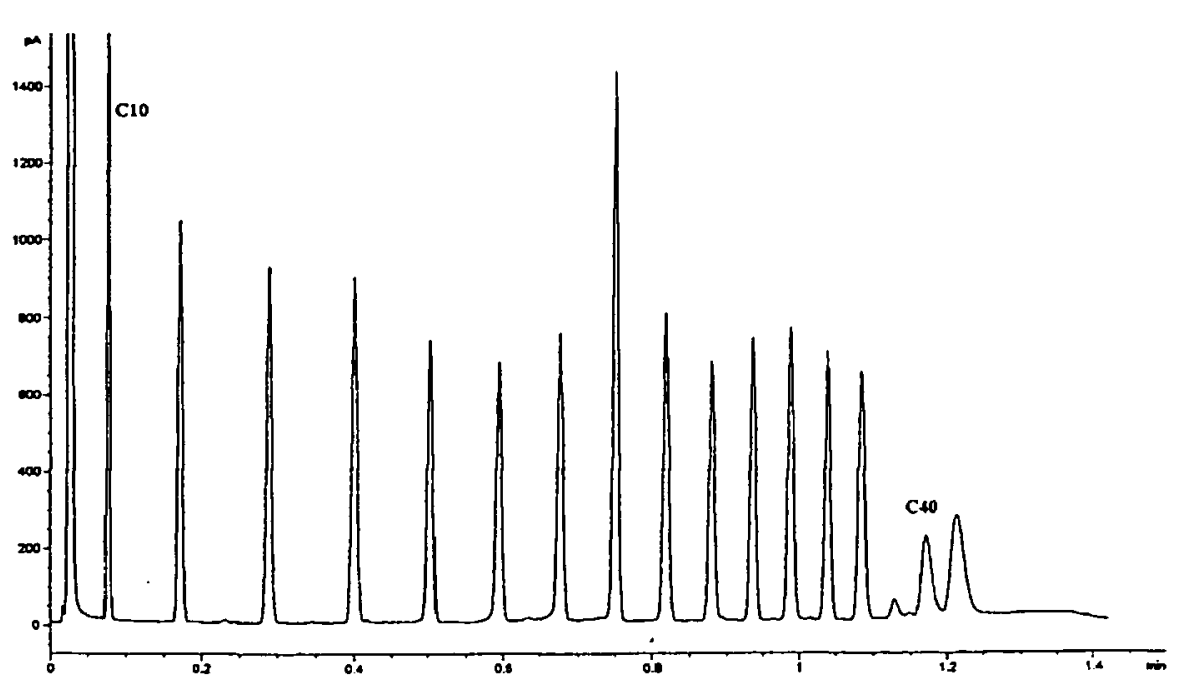

Figure 2 : Exemple d'analyse en Fast GC (chromatographie gazeuse ultra rapide). Analyse d'un mélange d'alcanes $\left(C_{10^{-}} C_{42}\right)$ en 90 secondes ; programmation par EZ flash 80 $375^{\circ} \mathrm{C}$ à $4^{\circ} \mathrm{C} / \mathrm{sec}$.

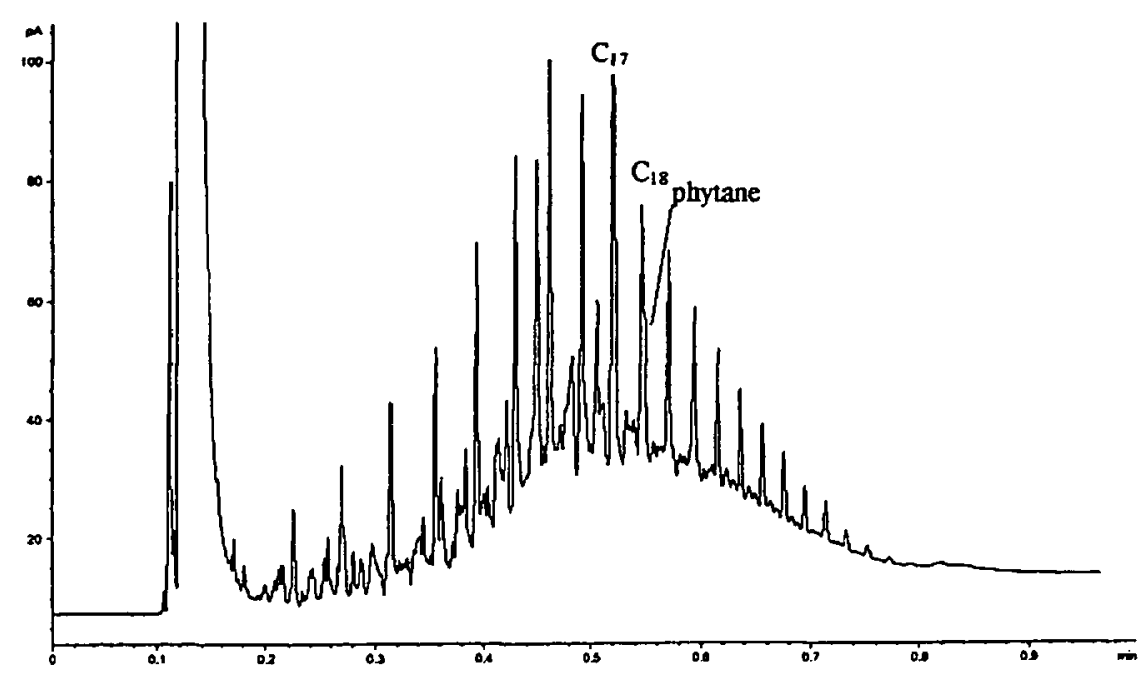

Figure 3 : Analyse d'un diesel dans l'heptane en 1 minute par chromatographie gazeuse ultra rapide. Programmation par EZ flash $60^{\circ} \mathrm{C}-350^{\circ} \mathrm{C}$ à $8^{\circ} \mathrm{C} / \mathrm{sec}$.

Le logiciel de verrouillage des temps de rétention (Hewlett Packard) exploite les possibilités du contrôle électronique de pression. Le temps de rétention d'un soluté donné (le composé de verrouillage) est relevé, et le logiciel établit la relation entre la pression et le temps de rétention : l'analyste peut alors utiliser un autre chromatographe. Il effectue l'analyse du composé de verrouillage, et entre la rétention de celui-ci dans le logiciel. Ce dernier calcule la pression permettant de faire correspondre le nouveau chromatogramme au chromatogramme obtenu précédemment.

La GC est très rapide, mais ne s'applique guère aux drogues ou autres agents toxiques qui sont métabolisés in vivo et donnent naissance à des composés polaires. La cocaïne, les opiacés, les amphétamines, etc. doivent être transformés en dérivés acétylés, fluorés, silylés ou être analysés en Chromatographie liquide (LC). L'avantage de la formation de dérivés est la détection sans ambiguïté en quelques secondes. Lorsque la séparation est très difficile et implique des composés de polarités très différentes, la solution la plus efficace est de recourir au couplage de deux chromatographies (gazeuse et liquide) ; c'est plus long et l'interface doit être soigné, mais les informations apportées sont nettement plus grandes.

Des progrès importants ont été réalisés ces dernières années dans le domaine des colonnes de chromatographie liquide HPLC. Les fabricants, poussés par les utilisateurs et par les normes, ISO 9000, EN 45001, les GLP (BPL) ont perfectionné la qualité et la reproductibilité. Si les particules de silice de $5 \mu \mathrm{m}$ sont d'usage courant, la tendance est l'emploi de particules plus petites ( $3 \mu \mathrm{m}$ et moins). On ne peut guère envisager de diminuer encore, car la perte de charge est proportionnelle au carré du diamètre des particules et devient 
alors rédhibitoire. C'est une des raisons (on verra l'autre plus loin) qui ont incité chercheurs et fabricants à se lancer dans la fabrication de monolithes. Il s'agit d'aérogels comportant deux types de pores : les uns sont relativement larges et sont analogues au volume extraparticulaire, les autres sont plus restreints et ressemblent aux pores intraparticulaires. La perméabilité très grande permettant des vitesses de liquide très élevées, des développements dans cette technologie devraient permettre un gain de temps considérable.

La micro HPLC permet d'économiser des solvants, car les colonnes capillaires de silice fondue ont un diamètre de l'ordre de 100 à $200 \mu \mathrm{m}$. Jusqu'à présent, la micro HPLC ne s'est guère implantée. Les choses risquent de changer avec le développement espéré de l'électrochromatographie ; c'est une technique hybride entre l'électrophorèse capillaire et la chromatographie. L'électrophorèse capillaire (CE) a connu un essor rapide et le nombre d'applications est allé croissant. Rappelons que la technique fait appel au flux electrosmotique engendré par un champ électrique. Le grand avantage réside dans le profil plat de vitesse par opposition au profil parabolique classique engendré dans un capillaire lorsque le liquide est poussé par une pompe. Un schéma de l'appareil (figure 4) montre bien la simplicité de l'équipement. La $\mathrm{CE}$ possède des avantages certains : grande efficacité exprimée en nombre de plateaux théoriques, colonnes de petite taille remplies de tampon (donc généralement pas de solvant, bien que les applications impliquant un solvant soient de plus en plus nombreuses), pas de pièces en mouvement (pas de pompes). Toutefois, on note sur la figure l'absence d'injecteur. L'introduction des solutés est, soit hydrodynamique soit électrocinétique, et implique un transfert du capillaire. En principe, seuls les solutés ionisables peuvent être séparés ainsi, mais le champ s'est élargi avec l'électrophorèse micellaire qui permet de traiter des solutés neutres.

En électrochromatographie, l'appareillage est identique à celui de l'électrophorèse capillaire, mais la colonne est remplie de particules fonctionnalisées (silice greffée RP18, par exemple). Il n'y a plus de pompe, puisque le liquide est entraîné par le flux électroosmotique. Les solutés sont séparés en fonction de leur mobilité électrophorétique et de leur partage entre phase solide stationnaire et phase liquide circulante. Nous en sommes aux débuts de cette technique, les séparations publiées sont très encourageantes et on fonde de grands espoirs à condition de régler les problèmes d'appareillage qui obèrent la validation des méthodes électrophorétiques en général.

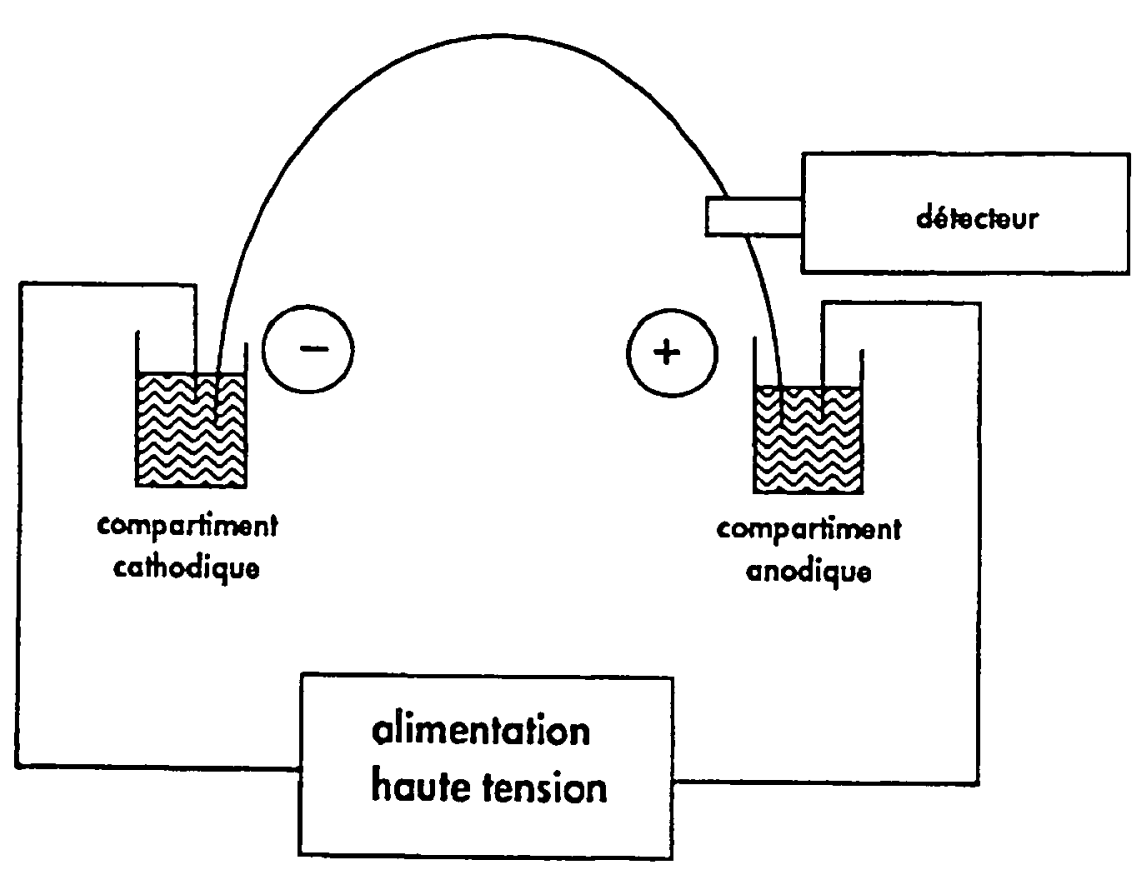

Figure 4: Schéma d'un appareillage d'électrophorèse capillaire.

Le maillon faible reste le traitement de l'échantillon. Là aussi, la miniaturisation est intervenue. Fini le temps des ampoules à décanter et des volumes de solvants à manipuler! L'extraction en phase solide (SPE) sur de petites cartouches de support solide, analogue à une phase de chromatographie, a remplacé ces opérations ennuyeuses. Les cartouches ont diminué de volume jusqu'à devenir des disques, et l'on fait percoler un ou deux millilitres d'échantillon en poussant avec une seringue, c'est encore trop ! La micro extraction (SPME) (2) n'utilise plus qu'une fibre absorbante rétractable et quelques microlitres de solution suffisent !

\section{Rien ne sert de séparer, il faut détecter.}

Le détecteur universel existe, c'est le spectromètre de masse. La GC/MS est devenue une technique de routine, tous les appareils à couplage GC/MS offrent la possibilité d'introduire directement l'échantillon au niveau du spectromètre de masse. Dans le cas de la chromatographie liquide ou de l'électrophorèse capillaire, deux types d'interface ont pris le pas sur les autres: l'electrospray (en français électronebulisation) et l'ionisation chimique à pression atmosphérique (APCI). Tous les couplages avec la masse reposent sur le principe de l'acquisition scannée. On divise le temps imparti à la séparation en "scans" au cours desquels on enregistre un spectre de masse. Plus le nombre de scans par minute est élevé, meilleure est la définition des pics. Cela implique une taille respectable du fichier informatique. Le couplage LC/MS peut poser quelques problèmes de constantes de temps. Un instrument de type quadripole 
nécessite 2 secondes pour acquérir un spectre dans la gamme $0-2000 \mathrm{~m} / \mathrm{z}$. La spectrométrie à temps de vol peut stocker 100 spectres à la seconde. Des constructeurs comme Sensar proposent des spectromètres à temps de vol capables de s'accomoder de pics dont la largeur est de 20 millisecondes.

Les analyseurs de masse à temps de vol sont en aval de la MALDI (Matrix Assisted Laser Desorption Ionization). Les ions formés dans la source sont accélérés jusqu'à énergie cinétique constante dans un tube d'entraînement (drift tube) et ils arrivent au détecteur au bout d'un temps proportionnel au carré de leur masse. Le temps de séjour est très bref (3-6 $\mu \mathrm{s})$; au début, ces appareils n'étaient pas très résolutifs. Ce n'est plus le cas maintenant et ils rivalisent avec les instruments à secteur. Ils sont miniaturisés pour atteindre seulement quelques centimètres. De plus, la gamme des $\mathrm{m} / \mathrm{z}$ est pratiquement illimitée. Au moyen de colonnes courtes et efficaces et de la détection MS/MS, on a pu réaliser l'analyse de six benzodiazepines de 1100 patients en 12 heures!

Si l'on avait dit il y a dix ans que l'on arriverait à détecter une seule molécule, on aurait pris cela pour une plaisanterie : c'est pourtant chose faite au moyen d'un laser qui illumine un capillaire. La molécule à détecter réagit avec une sonde et produit une fluorescence. Un filtre à vapeur métallique de rubidium absorbe la diffusion spéculaire du capillaire. La fluorescence des molécules peut être alors détectée. Le signal est mesuré durant le temps de transit d'une molécule à travers un volume de 1 picolitre et un filtre digital extrait le signal.

La fluorescence induite au laser (LIF pour Laser Induced Fluorecence) est actuellement le mode de détection le plus répandu en électrophorèse capillaire. Avec des dérivés fluorescents (cas des dérivés fluorenyl d'amino acides par exemple), des quantités de $10^{-18}$ moles sont détectables.

Le couplage de la chromatographie et de la RMN est une réalité. Il n'est pas possible de miniaturiser beaucoup l'appareil de RMN, mais l'effort a porté sur l'interface et surtout l'acquisition du signal. Les travaux du groupe de Tubingen (3) en Allemagne ont permis une avancée décisive dans ce domaine. On peut même, comme le fait Zare, utiliser un microscope confocal comme détecteur (4).

\section{Le portable}

Un effort considérable a porté sur les instruments portables. Le $\mathrm{pH}$ mètre a été un des premiers appareils miniaturisés et transportables. On peut le mettre dans la poche de la chemise et il n'y a plus de cordon. D'une manière générale, les appareils portables ont été élaborés pour répondre aux besoins analytiques liés à la dégradation de l'environnement (air, eau). Le spectrophotomètre infrarouge (proche IR) possède un câble contenant des fibres optiques et peut pénétrer dans les bouteilles, les sacs et tout autre orifice. Un appareil à transformée de Fourrier peut se mettre dans un attaché case et ne pèse pas plus de $9 \mathrm{~kg}$ ! On dispose de chromatographes en phase gazeuse opérationnels sur le terrain. Perkin Elmer avait ouvert la voie, et son Voyager a eu du succès. Inficon a ensuite lancé l'Hapsite (figure 5) qui est équipé d'un détecteur à spectrométrie de masse : l'ensemble n'est pas lourd, la batterie d'alimentation étant l'élément qui pèse le plus. On trouve maintenant sur le marché des portables équipés d'un espace de tête ou d'un échantillonneur ultra rapide. Les cosmonautes disposent d'un analyseur d'éléments par fluorescence $\mathrm{X}$ portable. La philosophie qui a conduit au lancement des portables est de ne plus "porter l'échantillon au labo". On réalise l'analyse sur place et on transmet par les moyens devenus classiques (Internet, Intranet etc.) les données au centre de décision. Si le GC/MS portable est une réalité, il n'en est pas de même de la LC ou de la CE car il est difficile de transporter un liquide surtout s'il s'agit d'un solvant.

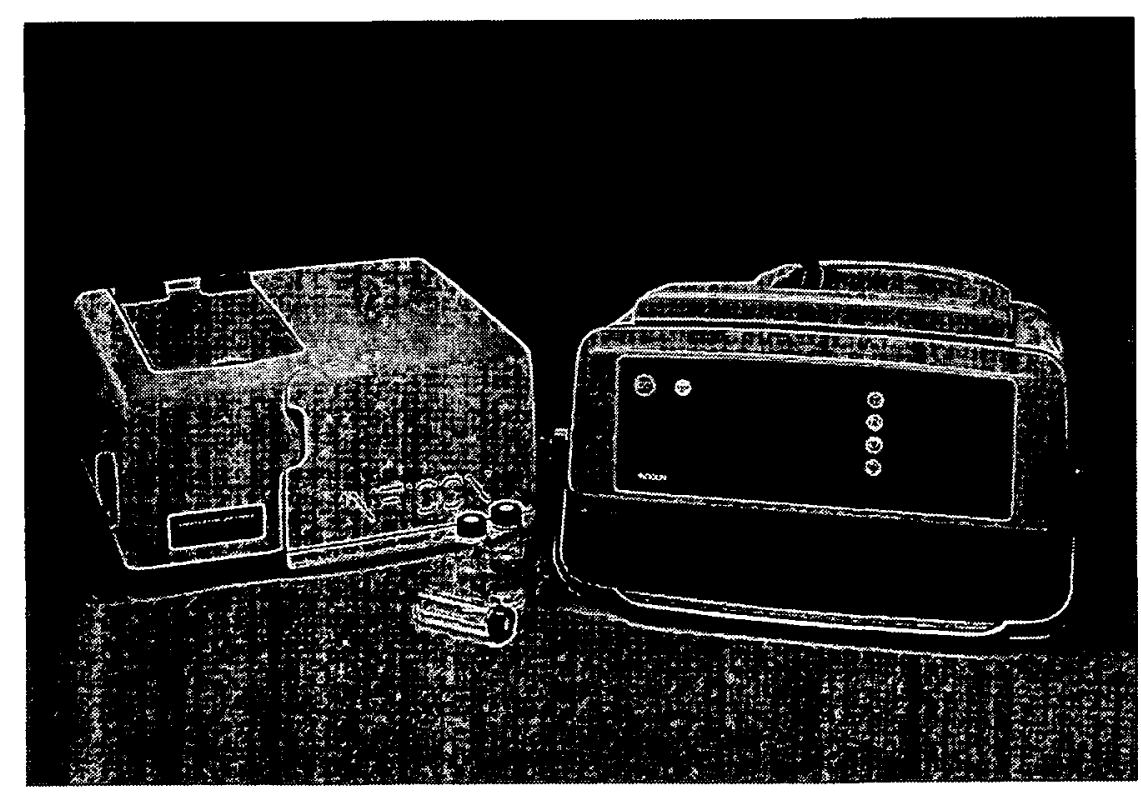

Figure 5 : Chromatographe en phase gazeuse portable (Inficon) à détection par spectrométrie de masse.

\section{De plus en plus petit : les puces.}

La miniaturisation des composants de l'électronique a eu des retombées sur l'instrumentation analytique. On est passé des colonnes "classiques" de chromatographie aux colonnes capillaires. Cela entraîne des écono- 
mies appréciables mais si les colonnes capillaires sont devenues courantes en GC, elles n'ont eu qu'un succès d'estime en LC.

Les besoins engendrés par l'étude du génôme, la chimie combinatoire, les neurosciences et l'étude des signaux chimiques transmis par les cellules ont conduit à la conception et au développement de systèmes analytiques gravés sur des puces. Les techniques de microlithographie sont bien maîtrisées puisque l'on produit des millions de transistors dont la taille est en dessous du micromètre. La fabrication de colonnes par microlithographie permet de réduire les dimensions et surtout de mettre sur un espace extrêmement réduit un nombre important de colonnes qui peuvent fonctionner en parallèle.

On est allé encore plus loin pour concevoir des unités capables de réaliser le traitement de l'échantillon par réaction chimique ou biochimique, la séparation des composés formés et leur détection : c'est la technologie dite des "Lab on chips". A.Manz (Imperial College, London,) a été le pionnier de l'electrophorèse sur chip (5 ) et J.M. Ramsey (Oak Ridge,USA) (6) a énormément développé la technologie en résolvant les difficultés. Une littérature abondante est parue. Les recherches dans ce domaine sont ciblées sur le séquençage de l'ADN, la défense contre les agressions chimiques ou biochimiques, la découverte de nouveaux médicaments. Un appareil est commercialement disponible, l'Agilent 2100, qui intègre sur une puce la gestion des fluides, le traitement de l'échantillon, la séparation et la détection. Trois kits d'essais sont disponibles : l'ADN 7500 pour l'évaluation précise de la composition et de la quantification des fragments d'ADN allant de $100 \mathrm{bp}$ à 7500 bp (paires de base), le 12000 et l'ARN 6000 pour l'ARN et l'ARNm. A présent, il est possible d'injecter des volumes de 1 picolitre sur des puces grâce à des microdoseurs. Le contrôle du débit de fluide est effectué en appliquant un voltage adéquat aux réservoirs (7). Le facteur critique est le croisement des canaux. Le grand avantage des microdébits est l'absence de turbulence et, par voie de conséquence le mélange est très rapide et la diffusion 100 fois plus rapide qu'avec un système 100 fois plus gros. La vitesse et la sensibilité des séparations électrophorétiques sur puce n'a cessé d'augmenter. Ramsey et coll. ont réalisé des séparations en moins d'une milliseconde ! On peut faire des immunoessais. Par exemple, Harrison (Université d'Alberta) intègre un immunoréacteur. Un échantillon de sérum dilué contenant le composé à identifier (la théophylline dans ce cas) est mélangé avec de la théophylline marquée par un traceur fluorescent et un anti- corps anti-théophylline. La théophylline libre entre en compétition avec la théophylline marquée. Après réaction, la solution est injectée dans le capillaire d'électrophorèse où se produit la séparation et la détection par fluorescence. Le même dispositif peut être monté dans une fibre optique. L'identification des protéines était une opération longue car elle impliquait de nombreuses étapes. Un microsystème complètement automatisé appelé $\mu$-chip IMER (Immobilized Enzyme Reactor) qui va du traitement de l'échantillon à la spectrométrie de masse par MALDI -TOF a vu le jour.

On réalise des faisceaux de canaux avec des géométries diverses, celui réalisé à Berkeley, USA étant particulièrement remarquable car on est arrivé à mettre jusqu'à 2000 canaux sur une lame de microscope.

La détection doit suivre. Il est indispensable de détecter des atomoles. Une interface de nanoelectronébulisation a vu le jour pour étudier par spectrométrie de masse des dérivés d'acides nucléiques. Un $\mu$ Chem Lab (portable évidemment) est en cours de développement chez Sandia. Il y aura échantillonnage, chromatographie (gaz ou liquide), détection, acquisition de données, communication des résultats. Les agents chimiques toxiques (le but est la lutte antiterroriste) sont détectés en 1 ou 2 minutes avec une limite de détection de l'ordre de la ppb.

Les puces à ADN sont des surfaces de verre (mais cela peut être aussi du silicium ou du nylon) sur lesquelles sont placés des fragments d'ADN par photolithographie. Sur une puce, on peut placer 65000 à 400000 oligonucléotides. ce qui représente 9000 gènes sur une surface de $1,6 \mathrm{~cm}^{2}$. Pour obtenir l'expression génétique, l'ARN messager est extrait du tissu à analyser, converti en ARN par une transcriptase qui incorpore des nucléotides fluorescents ou radioactifs dans l'ARN. L'ARN est incubé et hybridé. Les séquences hybridées sont détectées par fluorescence ou comptage. Les données sont stockées sous forme d'images digitalisées.

\section{La multidimensionnalité}

Mettre des colonnes en parallèle permet de faire des analyses simultanées mais n'augmente pas le pouvoir de séparation. Mettre des colonnes en série ne multiplie l'efficacité que par le nombre et l'on est vite limité par la longueur ou la pression. On sait que l'electrophorèse dite classique est capable de distinguer plusieurs dizaines de composés. Il est clair que réaliser deux types de séparations de façon orthogonale (comme c'est le cas en électrophorèse bidimensionnelle) ne multiplie pas l'efficacité par deux mais l'élève au carré. Il est 
indispensable que les deux types de séparations ne soient pas corrélés de façon à obtenir un espacement maximum des composés à séparer. Des essais prometteurs ont déjà été réalisés sur une simple plaque de chromatographie en couche mince mise sous pression. Ce n'est qu'un début et nous verrons sans doute de belles réalisations dans ce domaine. Pour que vienne le succès, il faudra résoudre les problèmes de détection. Les détecteurs actuels sont séquentiels et non simultanés. L'analyse d'image apportera certainement des solutions.

\section{Conclusion}

Nous n'avons considéré que les systèmes séparatifs et les techniques de détection qui y sont associées ou qui peuvent l'être. On peut mesurer la révolution produite par la miniaturisation.

Ceci n'est qu'un début. C'est la spectrométrie de masse qui a le plus "rétréci" et ce n'est pas fini. On travaille à réaliser des trappes d'ions sur une puce, on envisage de faire une batterie de trappes d'ions qui aurait l'aspect de tuyaux d'orgues en miniature, on travaille sur des alliages (les getters) pour remplacer les pompes. Tout cela va certainement amener à repenser le laboratoire avec une philosophie différente. On gardera à l'esprit que la miniaturisation entraîne forcément une perte de résolution et qu'un appareil portable est en général dédié à une application donnée. Il donne une indication rapide, permettant d'intervenir immédiatement.

Il est un aspect que nous n'avons pas évoqué, c'est celui de la validation de l'appareil et des données fournies. Là aussi, nous n'en sommes qu'au début.

\section{Références}

1. Van Deursen M, Janssen HG ,Beens J, Cramers CA. Strategies for speed optimization in GC. J.High Resolution Chromatogr.1999 ; $14: 184$.

2. Reisert R., Pawliszyn J. Design of automated solid phase microextraction for trace analysis of organic compounds in aqueous samples. J.Chromatogr A $1997 ; 776: 293$.

3. Gfroerer P., Schewitz J., Pusecker K., Bayer E. On line Coupling of Capillary separation Techniques with $1 \mathrm{H}$ NMR. Anal.Chem.1999; $71: 315$.

4. Zare $R$ N. A plunge in solution nanochemistry. Proceedings of the HPLC 1998 Symposium ,Saint Louis,MI, 12-15 juin 1998.

5. Manz A., Harrison D. J, Verpoorte E. M., Fettinger J.C., Paulus A., Widmer H.M. J.Chromatogr 1992 ; 593 : 253.

6. Khandurina J., Jacobson S.C., Waters L., Foote R.S., Ramsey J. M. Microfabricated porous Membrane structure for sample concentration and electrophoretic analysis. Anal .Chem. $1999 ; 71: 1815$.

7. Harrison J.D., Van den Berg A., Editors. Micro Total Analysis systems 98. Proceedings of the $\mu$ TAS 98 Workshop, Bauff, Canada, 1998 Kluwer Academic Publishers, Dordrecht, The Netherlands, 1998.

8. Ekstrom S., Onnerfjord P., Nilsson J., Bengtsson M., Laurell T., Marko-Varga G. Integrated Microanalytical Technology Enabling Rapid and automated protein Identification. Anal.Chem. $2000 ; 72: 286$. 